tion as part of a feedback loop, providing cues that trigger subsequent memories. Sniffing during olfaction is also a sequential activity, and it may have exactly the same function as eye movements during visual imagery-the activity itself may provide cues that trigger specific memories.

This line of reasoning may shed light on a fundamental question about imagery: why does the brain create mental images at all? Mental images are present for only a matter of seconds and must be created on the basis of information stored in long-term memory. Why should the brain go to the trouble of forming a mental image if the necessary information is already stored in long-term memory? Why not just access the stored information directly? One answer is that the long-term memory representations are not simply a copy of the representations that underlie short-term images, just as the code on a DVD is not simply a copy of the picture created on our computer or television screen. Representations differ in what information they make explicit and accessible ${ }^{4}$. The sniffs that occur in olfactory imagery trigger an internal representation that is then used to create the image. This image makes explicit and accessible the information that is only implicit and inaccessible in long-term memory. For example, imagine the smell of hot coffee or tea at the instant when you lift the mug to your lips and are just about to take a sip. Did you sniff? The perceptual information probably was stored in long-term memory in a concise format, as appears to be the case in visual memory ${ }^{5}$, and your sniff may have triggered a process that constructed an image on the basis of this stored information. You are then able to savor the scent once again. The fact that odors can be mentally mixed via olfactory imagery ${ }^{6}$ is consistent with this idea of forming 'odor images' by making information that is only implicit in long-term memory explicit and accessible. Indeed, Bensafi et al. ${ }^{3}$ suggest that sniffs may drive a pattern of neural activity in the cortex that underlies the odor image itself.

Neuroimaging studies of visual imagery have found that the first cortical areas to receive input from the eyes during perception (Brodmann areas 17 and 18) also are activated-even with eyes closed-when one visualizes objects with high resolution ${ }^{7}$. If these high-resolution details are not included in the image, only 'higher' visual cortex is activated. It would be fascinating to discover whether the same distinction exists in other sensory modalities. If true, this would suggest a general principle: when fine details of an object or event must be included in a mental image in order to perform a task, the brain can recruit the 'lowest' levels of neural processing - in both perceptual and motor systems.

\footnotetext{
. Kosslyn, S. M. Image and Mind (Harvard Univ. Press, Cambridge, Massachusetts, 1980).

2. Laeng, B. \& Teodorescu, D.-S. Cognit. Sci. 26, 207-231 (2002).

3. Bensafi, M. et al. Nat. Neurosci. 6, 1142-1144 (2003).

4. Marr, D. Vision (MIT Press, Cambridge, Massachusetts, 1982).

5. Tanaka, K. Annu. Rev. Neurosci. 19, 109-139 (1996).

6. Elmes, D.G. Chem. Senses 23, 443-445 (1998).

7. Kosslyn, S. M. \& Thompson, W. L. Psychol. Bull. 129, 723-746 (2003).
}

\title{
Pulling the Soxs off proliferation
}

A paper in this issue discloses the secret of eternal youth-at least for neural progenitors. During neurogenesis, self-renewing progenitor cells that reside in the ventricular zone of the developing CNS exit the cell cycle, migrate toward the marginal zone, and differentiate into neurons. Previous studies have identified transcription factors that promote the formation of new neurons by directing stem cells to stop dividing. However, the mechanisms responsible for countering differentiation to maintain a pool of undifferentiated neural progenitor cells have been less clear.

On pages 1162-1168, Jonas Muhr and colleagues report that the transcription factors Sox1, Sox2 and Sox 3 are important for maintaining the progenitor cell pool in the chick spinal cord. They find that Sox-3 maintains neural progenitor cells in an undifferentiated state and suppresses neuronal differentiation.

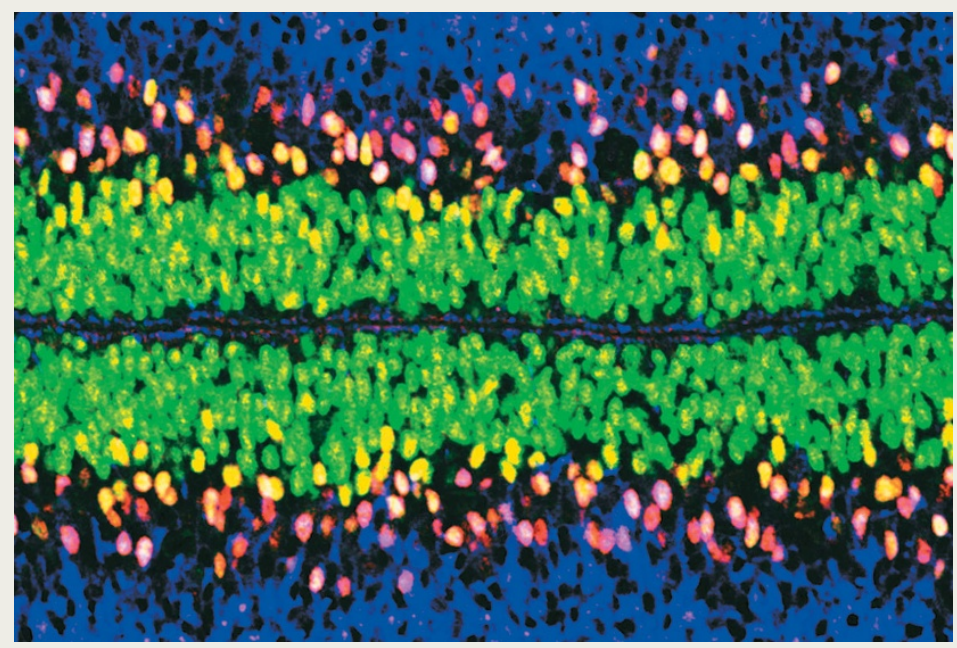
Sox $1-3$ proteins countered neurogenesis by repressing downstream differentiation signals from bHLH proteins, a family of proneural transcription factors. Moreover, when Sox1-3 target genes were actively repressed, independently of proneural activity, progenitor cells differentiated prematurely. The figure shows a coronal section of an embryonic chick spinal cord stained with Sox3 (green), NeuroM (red) and Tuj1 (blue). NeuroM is a bHLH protein, expressed during neurogenesis, whereas Tuj1 is a neuronal marker.

The Sox family of transcription factors are expressed by most stem cells in the developing CNS and are typically downregulated during differentiation. Sox genes are important in the generation of neuroblasts in Drosophila, and in maintaining precursor cells of the mouse blastocyst in a multipotent state. A Sox gene family member, Sox 10, expressed in migrating neural crest cells, maintains the multipotency of neural crest cells in culture. Moreover, Sox proteins are expressed in self-renewing stem cells in the adult CNS. Together with the work of Muhr and colleagues, these findings suggest that several Sox genes may keep cells from different origins in an undifferentiated state.

\section{Kalyani Narasimhan}

